\title{
Direct Conversion of Human Umbilical Cord Blood into Induced Neural Stem Cells with SOX2 and HMGA2
}

\author{
Jae-Jun Kim ${ }^{1,2, *}$, Ji-Hee Shin ${ }^{1,2, *}$, Kyung-Rok Yu ${ }^{1,2,3, *}$, Byung-Chul Lee ${ }^{1,2}$, Insung Kang ${ }^{1,2}$, Jin Young Lee ${ }^{1,2}$ \\ Da-Hyun Kim ${ }^{1,2}$, Yoojin Seo ${ }^{4,5}$, Hyung-Sik Kim ${ }^{4,5}$, Soon Won Choi ${ }^{1,2}$, Kyung-Sun Kang ${ }^{1,2}$ \\ ${ }^{1}$ Adult Stem Cell Research Center, College of Veterinary Medicine, Seoul National University, Seoul, Korea \\ ${ }^{2}$ The Research Institute for Veterinary Science, College of Veterinary Medicine, Seoul National University, Seoul, Korea \\ ${ }^{3}$ Hematology Branch, National Heart, Lung and Blood Institute, National Institutes of Health, Bethesda, USA \\ ${ }^{4}$ School of Medicine, Pusan National University, Busan, Korea \\ ${ }^{5}$ Biomedical Research Institute, Pusan National University Hospital, Busan, Korea
}

Recent advances have shown the direct reprogramming of mouse and human fibroblasts into induced neural stem cells (iNSCs) without passing through an intermediate pluripotent state. Thus, direct reprogramming strategy possibly provides a safe and homogeneous cellular platform. However, the applications of iNSCs for regenerative medicine are limited by the restricted availability of cell sources. Human umbilical cord blood (hUCB) cells hold great potential in that immunotyped hUCB units can be immediately obtained from public banks. Moreover, hUCB samples do not require invasive procedures during collection or an extensive culture period prior to reprogramming. We recently reported that somatic cells can be directly converted into iNSCs with high efficiency and a short turnaround time. Here, we describe the detailed method for the generation of iNSCs derived from hUCB (hUCB iNSCs) using the lineage-specific transcription factors SOX2 and HMGA2. The protocol for deriving iNSC-like colonies takes 1 2 weeks and establishment of homogenous hUCB iNSCs takes additional 2 weeks. Established hUCB iNSCs are clonally expandable and multipotent producing neurons and glia. Our study provides an accessible method for generating hUCB iNSCs, contributing development of in vitro neuropathological model systems.

Keywords: Human umbilical cord blood, Induced neural stem cells, Reprogramming, Direct conversion

Accepted for publication July 29, 2017, Published online November 30, 2017

Correspondence to Kyung-Sun Kang

Adult Stem Cell Research Center, College of Veterinary Medicine, Seoul National University, 1 Gwanak-ro, Gwanak-gu, Seoul 08826, Korea

Tel: +82-2-880-1246, Fax: +82-2-876-7610

E-mail: kangpub@snu.ac.kr

*These authors contributed equally to this work

(c) This is an open-access article distributed under the terms of the Creative Commons Attribution Non-Commercial License (http://creativecommons.org/ licenses/by-nc/4.0/), which permits unrestricted non-commercial use, distribution, and reproduction in any medium, provided the original work is properly cited.

Copyright (c) 2017 by the Korean Society for Stem Cells Research

\section{Introduction}

The advances in direct lineage conversion technology have facilitated the obtaining of diverse somatic cell types for regenerative medicine (1). Bypassing the pluripotent state, this technology could resolve the limitations of directed differentiation strategies using induced pluripotent stem cells (iPSCs). Several studies have reported that direct conversions into terminally committed cells such as neurons (2), hepatocytes (3) and cardiomyocytes (4) could complement the long-term differentiation and teratoma formation issues of iPSCs. However, these cell types inherently limit therapeutic applications because they are unable to proliferate. Thus, transdifferentiation into spe- 
cific adult stem or progenitor cells has proven to be advantageous for scalability and biomedical applications. Using this strategy, hepatic stem cells (5), hematopoietic progenitor cells (6), oligodendrocyte progenitor cells (7) and neural stem cells $(8,9)$ have been successfully induced from mouse and human somatic cells.

Previous studies have revealed that induced neural stem cells show robust capacities for unlimited proliferation and tri-lineage differentiation $(9,10)$. Additionally, when transplanted into mouse brain, the cells differentiated into neurons and glial cells, potentiating diverse in vivo applications. However, to date, the methods for generating iNSCs have met with limited success because of the tedious and inefficient reprogramming process required. Moreover, the starting cell sources were restricted to mouse somatic cells and human fibroblasts. Therefore, strategies for generating iNSCs from various cell sources and enhancing reprogramming efficiency are necessary.

In this context, human umbilical cord blood (hUCB) cells are an attractive alternative for use in reprogramming. The initial attempt, reported in 2009, demonstrated that hUCB cells are more amenable than other adult somatic cells for reprogramming into iPSCs (11). Moreover, the main advantages of hUCB are non-invasiveness for patients during acquirement and immediate availability with worldwide public bank (12). Additionally, these cells are in the immunologically naïve state, containing minimal genetic mutations compared with developmentally distinct cell types such as fibroblasts. Based on these characteristics, hUCB may be an attractive cell source for the generation of clinical-grade iNSCs.

Recently, Lee et al. reported that hUCB cells can be readily utilized for OCT4-mediated reprogramming into neural stem cells (13). Up to date, however, the detailed method for direct generation of SOX2-mediated iNSCs with hUCB cells has not been reported. We have previously reported that somatic cells can be directly converted into iNSCs with exogenous SOX2 and HMGA2 (14). Here, we presented SOX2-induced direct conversion method to reproducibly generate iNSCs from hUCB-derived $\mathrm{CD}_{3}{ }^{+}$cells without passing through pluripotent state. We also demonstrate that hUCB iNSCs have self-renewal and tripotent differentiation capacity.

\section{Materials and Methods}

\section{Human Umbilical cord blood CD34+ cell isolation and cell culture}

Mononuclear cells (MNCs) were harvested from hUCB using Lymphoprep (Stem Cell Technologies) density-gra- dient centrifugation. The isolation and research protocols were approved by the Boramae Hospital Institutional Review Board (IRB) and the IRB of Seoul National University (1109/001-006). Isolated mononuclear cells were subject to magnetic bead selection for purification of $\mathrm{CD}_{4}{ }^{+}$cells using a CD34 microbeads kit (Miltenyl Biotec) following the manufacturer's instructions. The purity of the isolated cells was assessed through flow cytometry. hUCB CD $34^{+}$cells were maintained in Iscove's modified Dulbecco's medium supplemented with $10 \%$ fetal bovine serum, $50 \mathrm{ng} / \mathrm{ml} \mathrm{SCF}, 100 \mathrm{ng} / \mathrm{ml}$ Flt3L, 50 $\mathrm{ng} / \mathrm{ml}$ TPO and $20 \mathrm{ng} / \mathrm{ml}$ IL-6 before infection. All cytokines were purchased from R\&D Systems.

\section{Flow cytometry analysis}

The cells were washed with PBS, incubated with anti-CD34-FITC antibody (BD Biosciences) for $30 \mathrm{~min}$ at $4^{\circ} \mathrm{C}$. After washing, cells were resuspended in $500 \mu 1$ of PBS and analyzed using FACSCalibur (BD Biosciences).

\section{Virus infection and neural stem cell culture}

Viral production and infection were performed as previously described (13). The retroviral $\mathrm{pMX}-\mathrm{SOX} 2$ and pMX-HMGA2 vectors were packaged in HEK293 FT cells (Invitrogen) using FuGENE 6 (Roche). hUCB CD34 ${ }^{+}$ cells were infected with retroviruses for 2 rounds in the hematopoietic cells growth medium. With day 0 designated as the time of first infection, the medium was changed to ReNcell NSC maintenance media (Millipore) containing $20 \mathrm{ng} / \mathrm{ml}$ bFGF and $20 \mathrm{ng} / \mathrm{ml}$ EGF on day 3 . All cytokines were purchased from R\&D Systems. As positive control for human neural stem cells, H9 hESC-derived NSCs (H9-NSCs, Invitrogen) were cultured using the ReNcell media.

\section{Immunocytochemistry}

Cells were fixed in $4 \%$ paraformaldehyde (PFA, in PBS) for $20 \mathrm{~min}$ at room temperature, permeabilized with $0.25 \%$ Triton X-100 for 10 minutes, incubated with 5\% normal goat serum for 1 hour and incubated with primary antibodies overnight at $4^{\circ} \mathrm{C}$. Subsequently, the cells were incubated with secondary Alexa 488- or 594-labeled antibodies (Invitrogen) for 1 hour at room temperature. The nuclei were counter-stained with 4',6-diamidino-2-phenylindole (DAPI) for 5 minutes. The images were taken using a confocal microscope (Eclipse TE200, Nikon). The details about primary antibodies are listed in Table 1.

\section{Quantitative reverse transcription-PCR (qRT-PCR)}

Total RNA was extracted using TRIzol reagent accord- 
Table 1. Antibodies used for immunocytochemistry

\begin{tabular}{cll}
\hline \multicolumn{1}{c}{ Primary antibody } & Manufacturer & Cat. no. \\
\hline $\begin{array}{ll}\text { Induced neural stem cells } \\
\text { Anti-SOX2 }\end{array}$ & Abcam & ab97959 \\
Anti-PAX6 & Covance & PRB-278P \\
Anti-HMGA2 & Abcam & ab52039 \\
Anti-Ki67 & Abcam & ab15580 \\
Anti-NESTIN & Abcam & ab22035 \\
Neurons & & \\
Anti-Neurofilament & Cell signaling & \#2836 \\
Astrocytes & & \\
Anti-GFAP & Abcam & ab4648 \\
Oligodendrocytes & & mab345 \\
Anti-O4 & Millipore & ab81093 \\
Anti-OLIG2 & Abcam & \\
\hline
\end{tabular}

ing to the manufacturer's instructions. cDNA was synthesized by reverse transcription using the Superscript III First-Strand Synthesis System (Invitrogen). qPCR was performed using the SYBR Green PCR Master Mix (Applied Biosystems). The following primers were used: SOX2 (F: AGTCTCCAAGCGACGAAAAA, R: TTTCACGTTTGCAACTGTCC), PAX6 (F: CAGCTCGGTGGTGTCTTTG, R: AGTCGCTACTCTCGGTTTA), NESTIN (F: AACAGCGACGGAGGTCTCTA，R: TTCTCTTGTCCCGCAGACTT), GLAST (F: CTCACAGTCACCGCTGTCAT, R: ACTATCTAGCGCCGCCATTC), OLIG2 (F: CCGATGACCTTTTTCTGCCG, R: CCACTGCCTCCTAGCTTGTC), OCT4 (F: GACAGGGGGAGGGGAGGAGCTAGG, R: CTTCCCTCCAACCAGTTGCCCCAAAC), NANOG (F: AATAACCTTGGCTGCCGTCTC, R: AGCCTCCCAATCCCAAACAAT).

\section{In vitro differentiation culture}

For differentiation and monolayer culture, hUCB iNSCs were seeded onto poly-L-ornithine/fibronectin (PLO/FN)-coated plates. Neural differentiation medium consisted of DMEM/F12 and Neurobasal medium (1:1), 2 mM GlutaMAX, 1\% Penicillin-streptomycin, $1 \mu$ M Retinoic acid, $20 \mathrm{ng} / \mathrm{ml} \mathrm{BDNF}, 20 \mathrm{ng} / \mathrm{ml}$ GDNF, $200 \mu \mathrm{M}$ Ascorbic acid, and $5 \mu \mathrm{M}$ Forskolin. For oligodendrocyte differentiation, cells were cultured in induction medium \#1 which consists of DMEM/F12, 1\% N2, 100x MEM-NEAA, $1 \mu \mathrm{M}$ Retinoic acid, $2 \mu \mathrm{M}$ purmorphamine, $2 \mathrm{mM}$ GlutaMAX and $10 \mathrm{ng} / \mathrm{ml}$ bFGF for 1 week. Subsequently, induction medium \#2 containing DMEM/F12, 0.5x N2, 2\% B27, 100x MEM-NEAA, $30 \mathrm{ng} / \mathrm{ml} \mathrm{T3,} 1 \mathrm{mM}$ cAMP, $2 \mu \mathrm{M}$ purmorphamine, $20 \mathrm{ng} / \mathrm{ml}$ PDGF, $100 \mathrm{ng} / \mathrm{ml} \mathrm{IGF}$, and $10 \mathrm{ng} / \mathrm{ml} \mathrm{NT3}$ was used for 2 weeks. For astrocyte differentiation, EGF and bFGF were removed from NSC maintenance medium and 1\% FBS was added.

\section{Results}

\section{Derivation of iNSC-like colonies from hUCB CD34 cells}

In order to generate iNSCs from hUCB, we first attempted to isolate MNCs from hUCB (Fig. 1A). As HetaSep sediments red blood cells (RBCs) by aggregation, HetaSep solution was mixed with hUCB samples at the ratio of 1:5. Subsequently, we applied Lymphoprep density gradient method to culture MNCs. After centrifugation, MNCs in between upper plasma and bottom Lymphoprep layers were harvested (Fig. 1B). We used typical magnetic bead for positive selection of $\mathrm{CD}_{3}{ }^{+}$cells from MNCs and purified cells were maintained in hematopoietic stem cells growth medium for 3 days (Fig. 1C). At day 1 of derivation, the purity was approximately 85 90\% (Fig. 1D). The conversion process was induced by two rounds of transduction of retroviral SOX2 and HMGA2 into hUCB CD34 ${ }^{+}$cells. We supplemented cytokines (SCF, Flt3L, TPO, and IL-6) in virus-containing medium to activate the proliferation of the population of hematopoietic stem cells, as retrovirus are transduced only into dividing cells. After the transduction, cells were cultured in IMDM with the cytokines for $24 \mathrm{~h}$ to further stimulate the proliferation. To re-plate suspended hUCB $\mathrm{CD}^{+}{ }^{+}$cells on feeder cells, we inactivated STO cells with mitomycin $\mathrm{C}$ and seeded them on gelatin-coated plates following a conventional feeder-dependent iPSC generation protocol (Fig. 1E). After 1 day, the medium was replaced with hNSC medium and replenished every day until iNSC-like colonies appear. We have found that although colonies appear as early as in 7 days, it is better to sustain the proliferation of colonies for more than 14 days for the ease of the colony-picking step (Fig. 1F and 1G). Finally, iNSC colonies were picked in small pieces using a pasture pipette and transferred to non-coated plates for homogeneous culture of neurospheres.

\section{Clonal expansion and characterization of hUCB iNSCs}

In order to determine whether the hUCB iNSCs have clonal expansion properties like human neural stem cells, we attempted to sub-culture neurospheres derived from hUCB iNSC colonies. The neurospheres derived from initial iNSC-like colonies were transferred on PLO/FN-coated plates for monolayer culture (Fig. $1 \mathrm{H}$ ). When the cells reached $80 \sim 90 \%$ confluence, they were detached using Accutase, and the neurosphere culture step was repeated at least three times for the establishment of homogeneous 
A

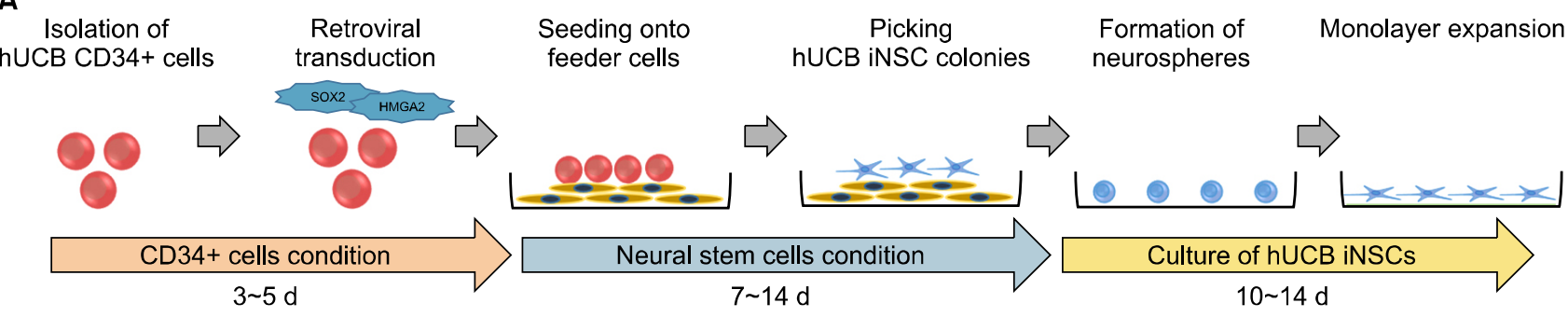

B

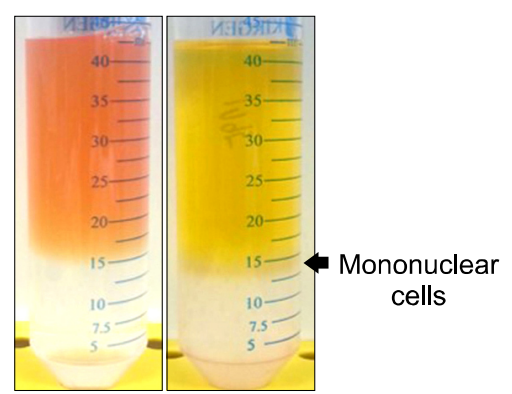

E

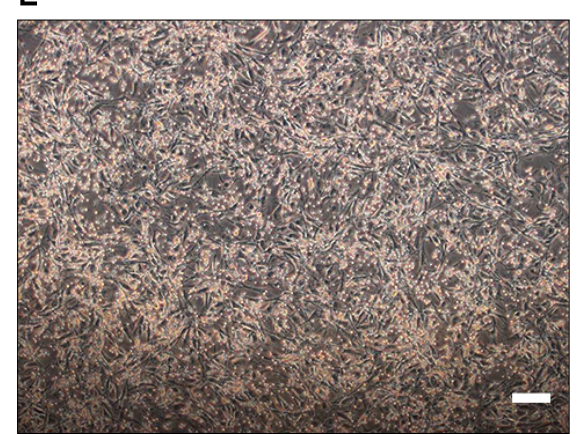

H

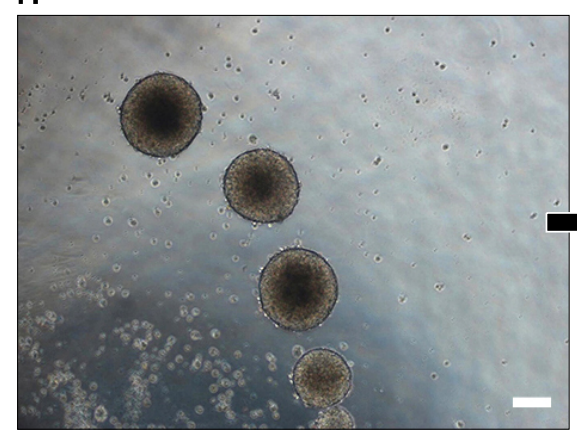

C

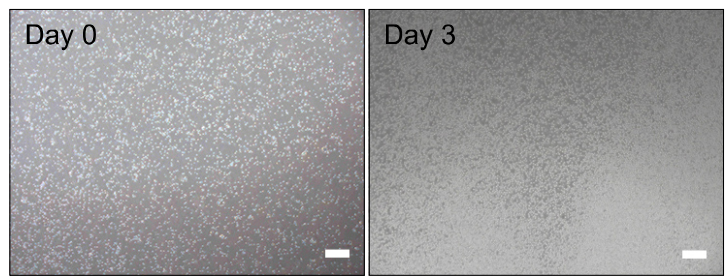

$\mathrm{F}$

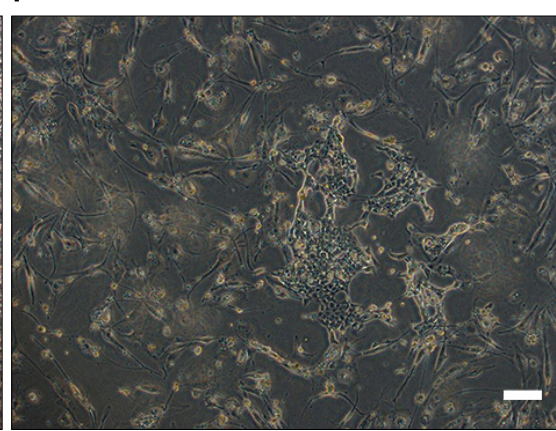

G
D

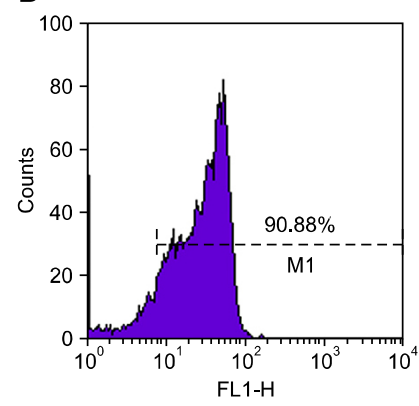

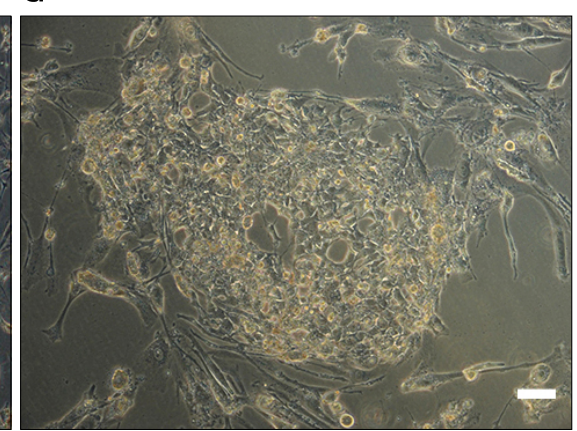

I
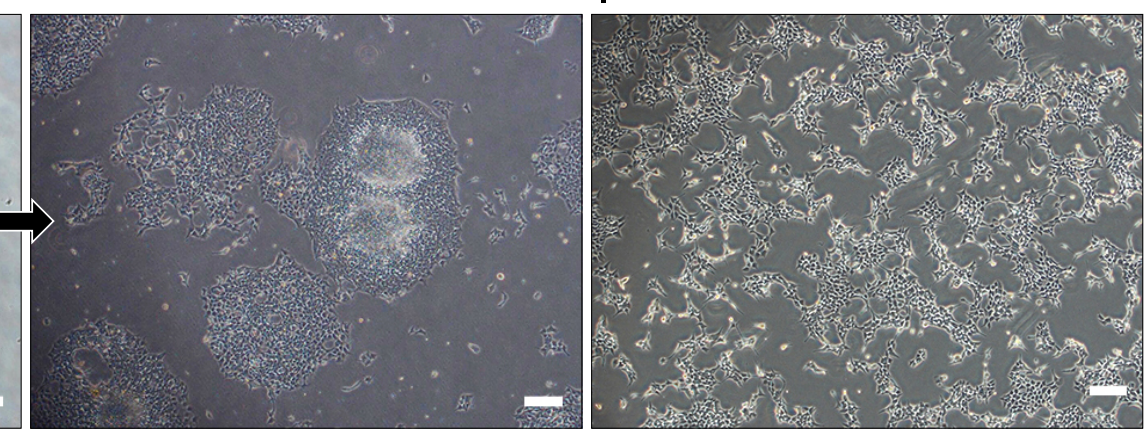

Fig. 1. Derivation of hUCB iNSCs. (A) Schematic presentation of protocols for hUCB iNSCs generation. hUCB CD34 ${ }^{+}$cells are isolated and expanded with proliferation-stimulating cytokines for $2 \sim 3 \mathrm{~d}$ and transduced with SOX2 and HMGA2 retrovirus. Distinct iNSC colonies appear after 7 10 d and are picked for repetitive sphere culture and monolayer culture for 10 14 d. (B) Before centrifugation, the upper layer is RBCs-reduced hUCB and the bottom layer is Lymphoprep (left). After centrifugation, a thin layer containing mononuclear cells appears between upper plasma layer and bottom Lymphoprep layer (right). (C) Phase contrast images of hUCB CD34 ${ }^{+}$cells cultured with cytokines at day 0 (left) and day 3 (right). Scale bars, $200 \mu \mathrm{m}$. (D) Flow cytometric analysis of hUCB CD34 $4^{+}$cells cultured for $1 \mathrm{~d}$ with cytokines. The cells were $90.88 \%$ positive for CD34. (E) Representative image of hUCB CD34 ${ }^{+}$cells seeded on STO feeder cells. $(F, G)$ Example of hUCB iNSC colonies cultured in neural stem cell inductive medium for $7 \mathrm{~d}(\mathrm{~F})$ and $12 \mathrm{~d}(\mathrm{G})$. (H) Morphology of neurospheres derived from initial hUCB iNSC colonies (left) and monolayer cultured hUCB iNSCs (right). (l) Morphology of homogeneous hUCB iNSCs cultured on PLO/FN-coated plates at passage 6. Scale bars in $(\mathrm{E}, \mathrm{F}, \mathrm{H}, \mathrm{I})$ and $(\mathrm{G})$ are $250 \mu \mathrm{m}$ and $100 \mu \mathrm{m}$, respectively. 
A

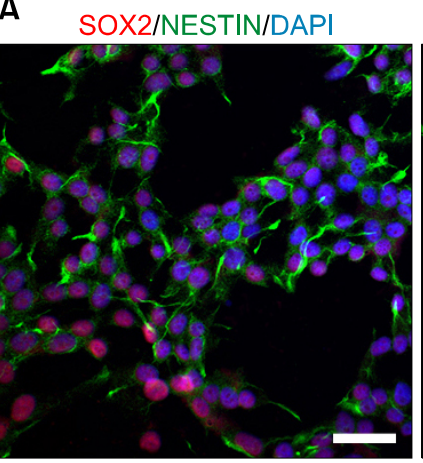

PAX6/NESTIN/DAPI

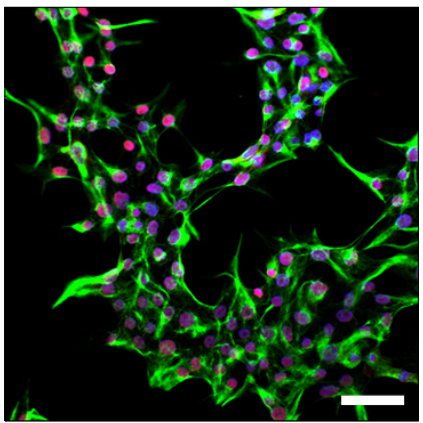

HMGA2/NESTIN/DAPI

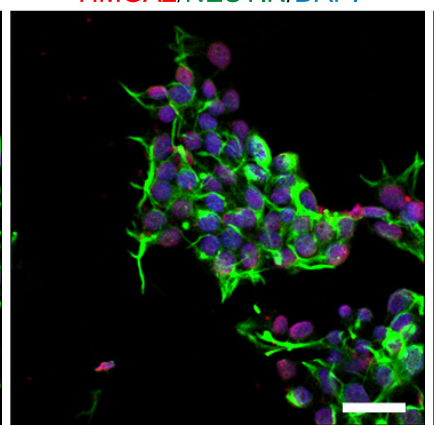

KI67/NESTIN/DAPI

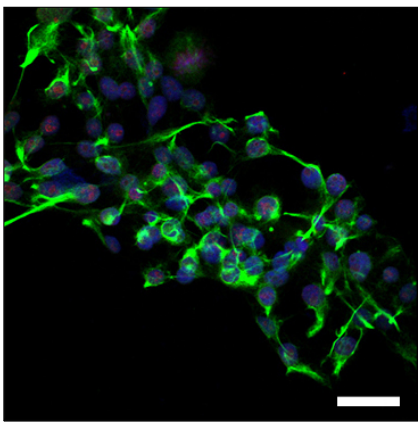

BLBP

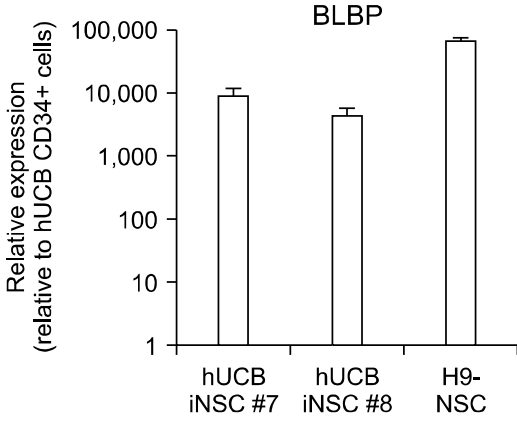

OLIG2

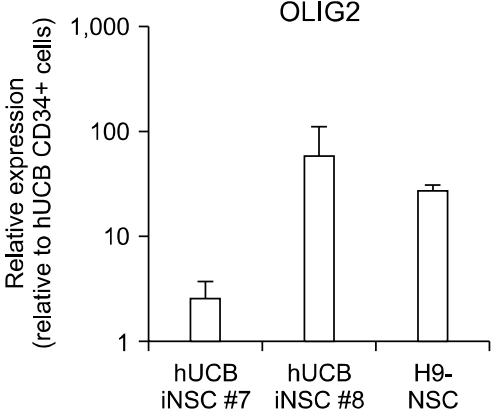

C

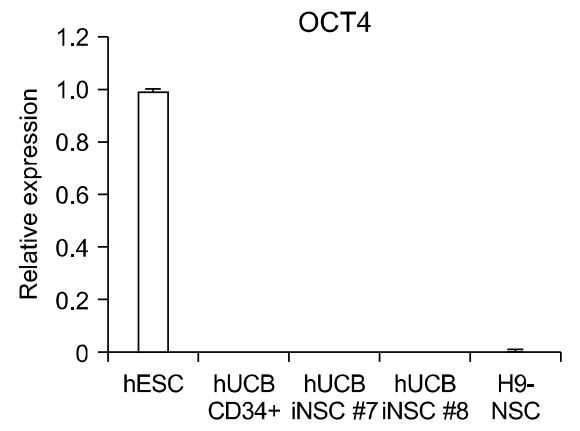

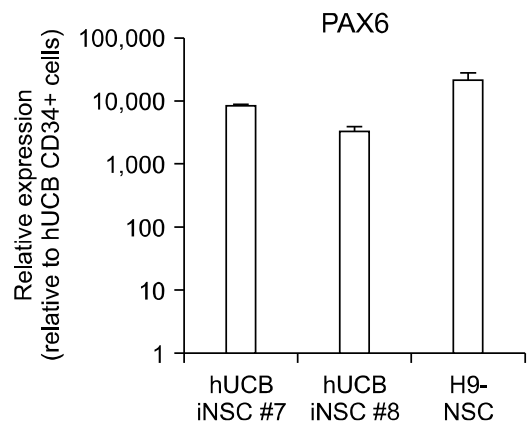

GLAST

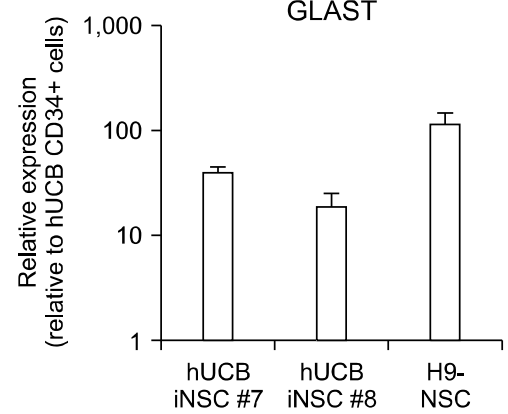

NANOG

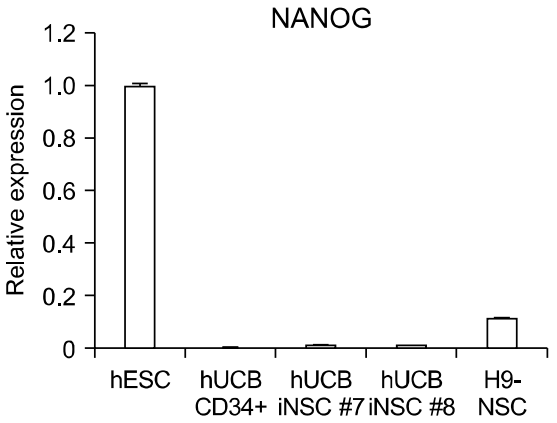

Fig. 2. Characterization of hUCB iNSCs. (A) Immunocytochemistry analysis of NSC-specific marker proteins in hUCB iNSCs using antibodies against SOX2, PAX6, NESTIN, HMGA2, and KI67. Nuclei were counterstained with DAPI. Scale bars, $50 \mu \mathrm{m}$. (B) Relative expression levels of the neural stem cell-specific markers were measured through qRT-PCR in two lines of hUCB iNSCs and H9-NSCs. (C) Relative expression levels of the pluripotent stem cell-specific markers were measured through qRT-PCR in human embryonic stem cell (hESC), hUCB CD34+ cells, two lines of hUCB iNSCs and H9-NSCs. Error bars denote the standard deviation of triplicate reactions. 

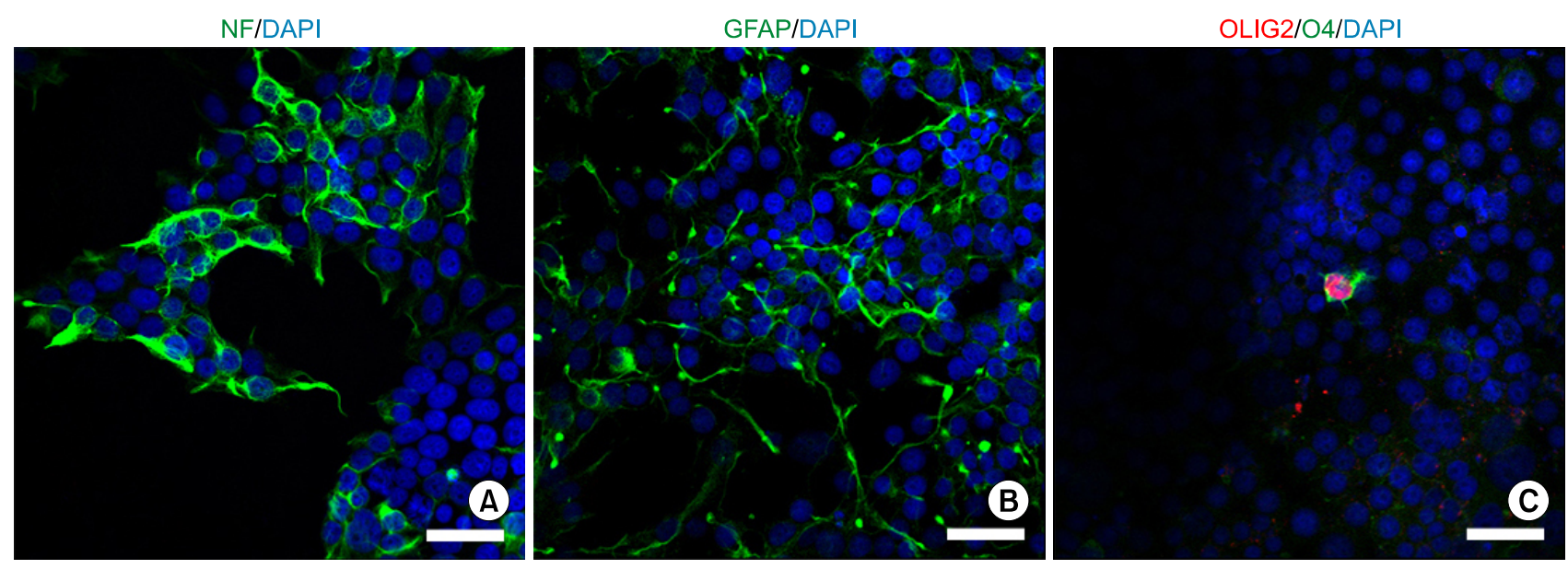

Fig. 3. In vitro differentiation of hUCB iNSCs. (A $\sim C$ ) Immunocytochemical analysis of hUCB iNSCs after differentiation into three major cell types: neurons (NF) (A); astrocytes (GFAP) (B); and oligodendrocytes (OLIG2 and O4) (C). Scale bars, $50 \mu \mathrm{m}$.

hUCB iNSC lines (Fig. 1I).

After passage 6, we briefly performed immunostaining with neural stem cell markers, PAX6 and NESTIN, for the selection of fully converted hUCB iNSCs. We have found that hUCB iNSC lines have different characteristics depending on their original colonies and that some cell lines show weak expression of neural stem cell markers. Upon passaging, immunostaining and qRT-PCR for diverse neural stem cell markers were performed, and activation of endogenous genes was confirmed before proceeding into further experiments. As shown in Fig. 2A, hUCB iNSCs prominently expressed neural stem cell-specific markers, including SOX2, NESTIN, and PAX6. Furthermore, hUCB iNSCs were immunoreactive for proliferation marker Ki67. qRT-PCR analysis showed that both two cell lines (hUCB iNSC \#7 and hUCB iNSC \#8) expressed endogenous NSC markers; SOX2, NESTIN, GLAST, BLBP, $P A X 6$, and OLIG2 (Fig. 2B). The expression levels of NSC markers were indistinguishable from H9-NSCs, suggesting that hUCB iNSCs were properly reprogrammed. Subsequently, we investigated whether the established hUCB iNSCs express pluripotent cell markers. qRT-PCR results showed that OCT4 and NANOG were not expressed in hUCB-iNSCs, suggesting that pluripotent cells are not contained in hUCB-iNSCs (Fig. 2C).

\section{hUCB iNSCs differentiate into tri-lineages}

To verify that the hUCB iNSCs were capable of differentiating into neurons, astrocytes and oligodendrocytes in vitro, we plated the cells onto PLO/FN-coated coverslips in 24 well culture plate. Withdrawal of bFGF and EGF and change into tri-lineage differentiation medium induced the differentiation of iNSCs. Accordingly, the cul- turing of hUCB iNSCs in neuronal differentiation media supported the generation of neurons, which expressed neuron-specific marker Neurofilament (NF) (Fig. 3A). Moreover, exposure of hUCB iNSCs to astrocyte differentiation media resulted in the formation of astrocytes that expressed GFAP (Fig. 3B). In addition, hUCB iNSCs could differentiate into O4- and OLIG2-positive oligodendrocytes (Fig. 3C). Thus, hUCB iNSCs are tripotent and can generate neurons, astrocytes, and oligodendrocytes in vitro.

\section{Discussion}

Our study shows that hUCB CD $34^{+}$cells can be robustly reprogrammed into iNSCs without pluripotency factors. These reprogrammed hUCB iNSCs are capable of clonal expansion and differentiation into tri-lineages.

Recently, several groups have reported methods for converting fibroblasts into iNSCs by the overexpression of pluripotency-associated factors under proper signaling environments $(9,15)$. However, such methods cannot eliminate the potential for passing cells through an intermediate pluripotent state during reprogramming, raising concerns for tumorigenesis even after establishment of iNSCs (16). In addition, a recent study reported that iNSCs generated using pluripotency factors show relatively high chromosomal instability (17).

On the other hand, Lujan et al. reported the direct conversion into iNSCs using lineage-specific transcription factors (18). In this method, expandable and multipotent iNSCs were generated from mouse somatic cells. However, this conversion method was limited by the requirement of multiple transcription factors and by very low efficiency. 
Subsequently, an advanced method was reported, transducing only the transcription factor SOX2 to convert mouse embryonic fibroblasts (MEFs) and human fetal foreskin fibroblasts (HFFs) into functional iNSCs (10). The conversion efficiency was improved, reaching $0.96 \%$ with MEFs when cultured on glass coverslips coated with poly-L-ornithine and laminin. However, this method was limited for its application to scalable experiments because seeding cells on glass coverslips was indispensable for inducing neural morphologies. Moreover, the conversion efficiency of HFFs described in this report remains unclear. The difficulty of obtaining human fetal skin biopsies could hinder further therapeutic applications.

In comparison with the outlined reports, we have broadened the cell sources to hUCB, which requires no invasive procedure and extensive culture prior to reprogramming. Moreover, we applied direct lineage conversion method which lacks the risk of tumorigenicity and we confirmed hUCB iNSCs lack expression of pluripotent markers.

However, our technology is accompanied by a weakness in the application for cell replacement therapy. We utilized retroviral vectors, which possess drawbacks such as genomic insertion and residual expression of transgenes. Therefore, the development of safer and efficient gene delivery methods is required for further clinical application. Recently, iPSCs were successfully generated with non-integrative gene-delivery tools such as mRNAs and proteins $(19,20)$. Moreover, the direct conversion of mouse somatic cells into iNSCs has been reported using cocktails of cell-permeable small molecules (21). Thus, it is expected that combinations of small molecules or non-integrative gene delivery methods will be applicable for the generation of hUCB iNSCs.

\section{Acknowledgments}

This study was supported by "Cooperative Research Program for Agriculture Science \& Technology Development (Project No. PJ01100201)" Rural Development Administration, Republic of Korea and partially supported by the Research Institute for Veterinary Science, Seoul National University (SNU, Republic of Korea).

\section{Potential conflict of interest}

The authors have no conflicting financial interest.

\section{References}

1. Xu J, Du Y, Deng H. Direct lineage reprogramming: strategies, mechanisms, and applications. Cell Stem Cell 2015;16:119-134
2. Vierbuchen T, Ostermeier A, Pang ZP, Kokubu Y, Südhof TC, Wernig M. Direct conversion of fibroblasts to functional neurons by defined factors. Nature 2010;463:10351041

3. Huang P, He Z, Ji S, Sun H, Xiang D, Liu C, Hu Y, Wang $\mathrm{X}$, Hui L. Induction of functional hepatocyte-like cells from mouse fibroblasts by defined factors. Nature 2011;475:386-389

4. Ieda $M, F u$ JD, Delgado-Olguin P, Vedantham V, Hayashi Y, Bruneau BG, Srivastava D. Direct reprogramming of fibroblasts into functional cardiomyocytes by defined factors. Cell 2010;142:375-386

5. Yu B, He ZY, You P, Han QW, Xiang D, Chen F, Wang MJ, Liu CC, Lin XW, Borjigin U, Zi XY, Li JX, Zhu HY, Li WL, Han CS, Wangensteen KJ, Shi Y, Hui LJ, Wang $\mathrm{X}, \mathrm{Hu}$ YP. Reprogramming fibroblasts into bipotential hepatic stem cells by defined factors. Cell Stem Cell 2013; 13:328-340

6. Riddell J, Gazit R, Garrison BS, Guo G, Saadatpour A, Mandal PK, Ebina W, Volchkov P, Yuan GC, Orkin SH, Rossi DJ. Reprogramming committed murine blood cells to induced hematopoietic stem cells with defined factors. Cell 2014;157:549-564

7. Yang $\mathrm{N}$, Zuchero JB, Ahlenius $\mathrm{H}$, Marro S, Ng YH, Vierbuchen T, Hawkins JS, Geissler R, Barres BA, Wernig M. Generation of oligodendroglial cells by direct lineage conversion. Nat Biotechnol 2013;31:434-439

8. Sheng C, Zheng Q, Wu J, Xu Z, Wang L, Li W, Zhang H, Zhao XY, Liu L, Wang Z, Guo C, Wu HJ, Liu Z, Wang L, He S, Wang XJ, Chen Z, Zhou Q. Direct reprogramming of Sertoli cells into multipotent neural stem cells by defined factors. Cell Res 2012;22:208-218

9. Thier M, Wörsdörfer P, Lakes YB, Gorris R, Herms S, Opitz T, Seiferling D, Quandel T, Hoffmann P, Nöthen MM, Brüstle O, Edenhofer F. Direct conversion of fibroblasts into stably expandable neural stem cells. Cell Stem Cell 2012;10:473-479

10. Ring KL, Tong LM, Balestra ME, Javier R, AndrewsZwilling Y, Li G, Walker D, Zhang WR, Kreitzer AC, Huang Y. Direct reprogramming of mouse and human fibroblasts into multipotent neural stem cells with a single factor. Cell Stem Cell 2012;11:100-109

11. Giorgetti A, Montserrat N, Aasen T, Gonzalez F, Rodríguez-Pizà I, Vassena R, Raya A, Boué S, Barrero MJ, Corbella BA, Torrabadella M, Veiga A, Izpisua Belmonte JC. Generation of induced pluripotent stem cells from human cord blood using OCT4 and SOX2. Cell Stem Cell 2009;5:353-357

12. Ballen KK, Gluckman E, Broxmeyer HE. Umbilical cord blood transplantation: the first 25 years and beyond. Blood 2013;122:491-498

13. Lee JH, Mitchell RR, McNicol JD, Shapovalova Z, Laronde S, Tanasijevic B, Milsom C, Casado F, Fiebig-Comyn A, Collins TJ, Singh KK, Bhatia M. Single transcription factor conversion of human blood fate to NPCs with CNS and PNS developmental capacity. Cell Rep 2015;11:1367-1376 
14. Yu KR, Shin JH, Kim JJ, Koog MG, Lee JY, Choi SW, Kim HS, Seo Y, Lee S, Shin TH, Jee MK, Kim DW, Jung SJ, Shin S, Han DW, Kang KS. Rapid and Efficient Direct Conversion of Human Adult Somatic Cells into Neural Stem Cells by HMGA2/let-7b. Cell Rep 2015;10:441-452

15. Zhu S, Wang H, Ding S. Reprogramming fibroblasts toward cardiomyocytes, neural stem cells and hepatocytes by cell activation and signaling-directed lineage conversion. Nat Protoc 2015;10:959-973

16. Bar-Nur O, Verheul C, Sommer AG, Brumbaugh J, Schwarz BA, Lipchina I, Huebner AJ, Mostoslavsky G, Hochedlinger $\mathrm{K}$. Lineage conversion induced by pluripotency factors involves transient passage through an iPSC stage. Nat Biotechnol 2015;33:761-768

17. Weissbein U, Ben-David U, Benvenisty N. Virtual karyotyping reveals greater chromosomal stability in neural cells derived by transdifferentiation than those from stem cells. Cell Stem Cell 2014;15:687-691

18. Lujan E, Chanda S, Ahlenius H, Südhof TC, Wernig M. Direct conversion of mouse fibroblasts to self-renewing, tripotent neural precursor cells. Proc Natl Acad Sci U S A 2012;109:2527-2532

19. Kim D, Kim CH, Moon JI, Chung YG, Chang MY, Han BS, Ko S, Yang E, Cha KY, Lanza R, Kim KS. Generation of human induced pluripotent stem cells by direct delivery of reprogramming proteins. Cell Stem Cell 2009;4:472-476

20. Mandal PK, Rossi DJ. Reprogramming human fibroblasts to pluripotency using modified mRNA. Nat Protoc 2013; 8:568-582

21. Zhang M, Lin YH, Sun YJ, Zhu S, Zheng J, Liu K, Cao $\mathrm{N}$, Li K, Huang Y, Ding S. Pharmacological reprogramming of fibroblasts into neural stem cells by signaling-directed transcriptional activation. Cell Stem Cell 2016;18:653-667 